\title{
An optimized segmentation and quantification approach in microvascular imaging for OCTA-based fibrovascular regression monitoring
}

\section{Sheng Wu}

The general hospital of People's Liberation Army

\section{Shaowei Wu}

Nanjing University of Science and Technology

Hui Feng

Zhongda Hospital Affiliated to Southeast University

\section{Zizhong Hu}

The first affiliated hospital of nanjing medical university

\section{Yejing Xie}

Nanjing Tech University

\section{Yun Su}

The first affiliated hospital of nanjing medical university

\section{Ting Feng ( $\nabla$ fengting@njust.edu.cn )}

Nanjing University of Science and Technology https://orcid.org/0000-0002-8676-1470

\section{Li Li}

Nanjing University of Science and Technology

\section{Research}

Keywords: OCTA, blood vessel segmentation, vessel extraction, neovascularization

Posted Date: July 20th, 2020

DOl: https://doi.org/10.21203/rs.3.rs-42242/v1

License: (c) (1) This work is licensed under a Creative Commons Attribution 4.0 International License. Read Full License 


\section{Abstract \\ Background:}

Quantification of neovascularization changes in fibrovascular membrane (FVM) acquired from optical coherence tomography angiography (OCTA) imaging is extremely important for diagnosis and treatment monitoring of proliferative diabetic retinopathy (PDR). However, few vessel extraction methods have been reported for quantifying the neovascular changes on fibrovascular membrane (FVM) in proliferative diabetic retinopathy PDR based on OCTA imaging.

\section{Results:}

The proposed method has achieved better performance compared with existing algorithms on accuracy and can be used for PRD treatment monitoring. In the study of PDR treatment monitoring, the data show that from the beginning ( 0 day) to 5th day of treatment, the total length of neovascularization on FVM in this area has been significantly shortened by an $77.8 \%$ reduction, indicating significant effects from the treatment applied. Besides, the average width of the neovascularization on FVM at the 7th day after treatment has been increased by $158 \%$, which indicates that most of the narrow neovascularization has been reduced.

\section{Conclusion:}

The result and analysis have confirmed that the proposed optimization process with improved VCA method is both effective and feasible to segment and quantify the neovascularization on FVM with less noise and artifacts, thus can be readily applied to monitor the fibrovascular regression within the treatment period.

\section{Background}

Proliferative diabetic retinopathy (PDR), characterized by neovascularization and fibrous hyperplasia, is a common and serious com diabetes plication of diabetes mellitus (DM) ${ }^{1}$. Persistent vitreous hemorrhage (VH) caused by neovascularization and tractive retinal detachment (TRD) may cause permanent vision loss or blindness, which is the most common indication for surgical intervention. Thus, clear visualization of neovascularization on the fibrovascular membrane (FVM) is critically important in diagnosis and treatment monitoring of PDR.

Efficient technologies to quantify neovascular changes on FVM in PDR are highly sought after, however, effective choices are fairly limited to date. The traditional color fundus photography can offer a certain amount of information, but its low resolution limits the ability to provide precise quantification ${ }^{2}$. The current "golden standard" to detect diabetic retinopathy is a dilated fundus examination with additional 
fluorescein angiography (FA) utilized to evaluate retinal vasculature in diabetic eye disease ${ }^{3,4}$. FA has high sensitivity in detecting microaneurysms and areas of neovascularization, which can be beneficial to visualize neovascular changes on FVM in diabetic eyes otherwise unobservable for examination. However, the FA method is unable to independently visualize the deep retinal capillary plexus.

In recent years, OCT angiography (OCTA), which is based on depth-resolved motion-contrast imaging, has emerged as a noninvasive imaging modality to image vasculars by comparing fluctuations in signal amplitude caused by relative movement of blood cells to static surrounding tissues ${ }^{5-7}$. Comparing with the traditional dye-based angiography, OCTA image acquisition is much more desirable as information on retinal and choroidal circulation can be almost instantly obtained with higher resolution. Over the past decade, substantial research effort has been dedicated to study OCTA-based microvascular changes on patients with diabetic retinopathy ${ }^{7,8}$. Quantitative studies analyzing retinal microvasculature with automated algorithms applied to OCTA have reported reduced vessel density in eyes with diabetic retinopathy ${ }^{6,9,10}$. Besides adaptive thresholding on OCTA, other techniques based on Hessian filters have also been proposed in animal studies ${ }^{11,12}$, which have revealed that the estimated vessel radius is very sensitive to the pre-selected value of maximum scale. To lessen the sensitivity of segmentation technique to Hessian filters' maximum scale parameter, another segmentation technique, which is based on intensity value, can be utilized in parallel to the Hessian technique with final segmentation achieved by compounding results. One of the artifacts observed in all OCTA approaches is the elongated vessels or tail artifact for which the vessel cross-section is in noncircular line-like shape ${ }^{13}$. The reason behind is that the connective eye tissues cause background light and dark changes in imaging, which interferes blood vessel recognition. Thus, rapidly fluctuating speckle signals below vessels will induce shadow-like artifacts in the angiogram data ${ }^{14}$. Due to such limitations, most vessel extraction algorithms to segment blood vessels are ineffective to segment the OCTA images. The most recent automatic quantification of vessel densities in OCTA images has explored the deep learning method ${ }^{15}$, however, it requires a large amount of OCTA images that are hard to collect within a short period of time.

In this study, we propose an optimized approach to segment blood vessels based on an improved vascular connectivity analysis (VCA) algorithm with a combined effort of morphological characterization as well as noise and artifacts elimination. The length and width of vessels are also obtained in quantitative assessment of the microvascular network. Furthermore, we also present a feasibility study of the proposed method by monitoring changes of neovascularization on FVM, which is based on the OCTA images of PDR patients after treatment by intravitreal injection of conbercept (IVC).

\section{Results}

\subsection{OCTA image acquisition}

The OCTA images collected by AngioVue are shown in Fig. 1. As observed from the original OCTA image, Fig. 1(a), it exists at least one artifact with a substantial noise level resulting in a fairly low signal to noise 
ratio $(<50)$. The vascular network extracted by the traditional matched filtering method ${ }^{16,17}$ is shown in Fig. 1(b). Utilizing the blood vessel network and morphological operation, the blood vessel skeleton is extracted and shown in Fig. 1 (c). It is observed that the matched filtering algorithm can maintain the continuity of blood vessels and obtain a clear blood vessel skeleton pattern. However, the extracted blood vessels obtained by this traditional matched filtering method are thicker than real blood vessels, which can directly alter the accuracy of quantitative blood vessel thickness. In addition, due to the high image noise level, the matched filter method cannot completely eliminate the artifact and image noise. The extracted blood vessel network is thus longer than the actual blood vessel, thus deteriorates the accuracy for both the blood vessel thickness and length.

\subsection{The optimization process}

Here, the blood vessel of Fig. 1(a) has been extracted and quantified again utilizing the improved VCA method proposed in Sect. 2.3. Figure 2(a) shows the vascular network extracted through the improved VCA method. In addition, to obtain the length and thickness of blood vessels, we have performed a binarization process on the image in Fig. 2(a) with results shown in Fig. 2(b). By using the skeleton extraction algorithm, the skeleton of blood vessel network shown in Fig. 2(b) has been obtained with results shown in Fig. 2(c). Comparing Fig. 2(c) with the original image of Fig. 1(a), it is clearly observed that the vascular network is now completely extracted. However, some noise clusters near the blood vessel are still mistakenly identified as blood vessels, such as the one marked by a yellow circle shown in Fig. 2(c). Thus, the blood vessel analysis method based on piece-by-piece analysis is required for further cleansing these artifacts. The final optimized image after all processes is shown in Fig. 2(d) and the vascular network is completely extracted and clarified with all artifacts and noise effectively removed.

\subsection{Treatment monitoring of the neovascularization in PDR}

As a feasibility study of our proposed optimization method in segmentation and quantification of morphological changes of neovascularization on FVM, we have applied this method in treatment monitoring of PDR. The OCTA images of a patient with PDR are shown in Fig. 3. Figure 3(a) illustrates distribution of the neovascularization on FVM of the patient before treatment, and Fig. 3(b)-(e) illustrate the OCTA images of the same region of the patient at different stages of treatment $(1,3,5,7$ days respectively). The optimized VCA algorithm has been used to extract the complete proliferating membrane vascular network, and the resulting binarized OCTA images as well as the vascular skeleton are shown in Fig. $3(\mathrm{f}-\mathrm{j})$ and Fig. $3(\mathrm{k}-\mathrm{o})$, respectively. It is clearly indicated from the blood vessel extraction results that distribution range of blood vessels decreases significantly with accumulated days of treatment. To evaluate variation in vascular distribution and thickness objectively, the length and average width of blood vessels in vessel network have been quantified, as shown in Fig. 4(a) and (b).

The thus obtained data show that from the beginning ( 0 day) to 5 th day of treatment, the total length of neovascularization on FVM in this area has been significantly shortened by an 1894 px reduction, indicating significant effects from the treatment applied. From the 5th to 7th day, the total length of blood vessels has continued to decrease by an $841 \mathrm{px}$ reduction. Overall, the length of neovascularization on 
FVM has been reduced by a total of $1955 \mathrm{px}$ in the treatment period, which is $77.8 \%$ less than that of pretreatment. Besides, as shown in the Fig. 4(c), the average width of the neovascularization on FVM from the beginning ( 0 day) to the 7 th day of treatment has been increased by $1.7 \mathrm{px}$, which is $158 \%$ larger than that of pre-treatment.

\section{Discussion}

Comparing with results from the traditional matched filter method shown in Fig. 1(b) and (c), our optimized VCA method can efficiently remove a large amount of noise scattered around the periphery and get clean vascular network on FVM shown in the OCTA images. In addition, the improved VCA method can effectively reduce the near-point noise and completely avoid the artificial noise, resulting in a much more accurate vascular length and width measurement. Besides, in the feasibility study of improved VCA method in monitoring changes of neovascularization on FVM, it shows that the neovascularization is mostly eliminated after the treatment period. Furthermore, the quantified results show that the length of microvascular was markedly reduced after treatment, while the mean width of vessels was increased due to the reduction of microvascular. The quantified result from image analysis is consistent with the clinical medical observation and diagnosis, which has strongly proved the effectiveness of improved VCA. Therefore, the above results have indicated that our optimized VCA and morphological characterization method can be effectively applied to quantify and monitor the changes in microvascular on FVM with OCTA images.

One of the main limitations of this study is the noise and artifacts reduction method which based on piece-by-piece analysis needs a threshold value to identify the noise and artifacts from the OCTA images, and may be not suitable for the images with multiple kinds of noise and artifacts. Furthermore, the improved VCA algorithm needs to search the starting points of the microvascular in the OCTA images from different directions, which leads to computing redundancy and should be further improved. In addition, studies involving larger number of samples from patients are necessary to verify the improved VCA algorithm in the future.

\section{Conclusion}

In summary, an optimized image segmentation and quantification approach, by combining an improved VCA algorithm with the morphological characterization method, has been proposed and demonstrated as effective and feasible for extraction of vascular network from OCTA images that are acquired from FVM of patients with PDR. Comparing with the traditional matched filter method, the optimized VCA method can achieve a final vascular network with lower noise and better robustness. Furthermore, our study also shows that the improved VCA algorithm can be practically applied to monitor the changes of neovascularization on FVM within treatment duration.

\section{Materials And Methods}




\subsection{Subjects}

This is a single-center prospective, comparative, observational clinical trial conducted in the First Affiliated Hospital of Nanjing Medical University. The trial has been registered with the Chinese Clinical Trial Registry (http://www.chictr.org/cn/ registration number: ChiCTR-IPR-17014160). The study has followed the tenets of the Declaration of Helsinki, and the study protocol has been approved by the Ethics Committee of First Affiliated Hospital of Nanjing Medical University (2015-SR-150). Informed written consents have been obtained from all patients after explaining possible consequences of the study. For this initial study, the data acquired from two human subjects in vivo have been analyzed in this paper.

\subsection{The OCTA Imaging}

For the OCTA imaging, AngioVue (version 2017.1.0.151; Optovue, Fremont, CA, USA) has been used to obtain split-spectrum amplitude decorrelation angiography. Two trained examiners (YS and LC) have performed the OCTA examinations after pupil dilatation using the incorporated B-follow-up eye-tracking model. The scanning area is centered on the FVM arising from the optic disc in $6 \times 6 \mathrm{~mm}$ sections. The navigation line is dragged between retina and FVM, followed by setting the Bvitreous as reference to exclude retinal vessels underneath. Manual adjustment is occasionally needed to segment retina and FVM on the B-scan model. Low-quality images with signal strength index $<50$, images with severe artifacts due to poor fixation, or undetectable images owing to FVM floating too high in the vitreous have been excluded in the analysis. All OCTA examinations have been performed three times for the mean values.

\subsection{OCTA microvascular extraction and quantification}

Based on characteristics of the OCTA blood vessels, an improved blood vessel extraction and quantification method utilizing the VCA method is proposed, which is composed of three parts including (1) pre-processing, (2) vessel extraction, and (3) vessel quantification. Figure 1 illustrates the three parts and the detailed operations in each step. For each OCTA microvascular image to be processed, a binary image that shows the microvascular of input image with quantified parameters of the microvasculars will be obtained after applying the various processing operations. The first pre-processing part includes image cropping and color space conversion. The next vessel extraction part includes operations of starting points detecting, vascular networks searching, binarization by automatic thresholding OSTU method, skeleton extraction of blood vessels, artifacts elimination, and vascular network merging. The final vessel quantification part includes quantification of the length and width. The image processing software used in this study is MATLAB (version R2016a; MathWorks, Inc., Natick, MA, USA). The detailed operations in each part are described as following.

In the first pre-processing part, the input image is processed by a two-step process to ensure the subsequent operations simple and effective. The original image is cropped to select the region of interest $(\mathrm{ROI})$, and then the color space is transformed to gray domain to reduce the computational complexity. 
In the second part, the VCA method is applied to determine the connected area from starting points of blood vessels as the microvascular network. In order to acquire the accurate and optimized vascular network sets with low noise and artifacts, an improved VCA method is hereby proposed. The main three processing steps in the second part are listed in the following.

The first step is to identify the starting points of vascular network. The matrices of OCTA images are traversed by performing the partial line detection, and the number of lines with no effective points are recorded. If the proportion of the value is lower than the preset threshold, this point is considered as a starting point. Then, the Z-shaped traversal is performed and continued to search other starting points of qualified vascular network until all traversal is completed.

The second step is to search all vascular networks connected with starting points. In the beginning, all starting points are marked as part of initial vascular network and restored in the network point set. Then, we move the detection coordinate from starting points to the next position in the OCTA image, and calculate the minimum distance between the detected point to all points in the network point set. If is small enough (within 2 pixels), which means the detection point is close enough or connected with some vessels restored in the network point set, it will be marked as part of the initial vascular network and stored in the network point set. Through the global image traversal, all point sets that meet the requirements are stored and the initial blood vessel network is thus obtained. However, since the blood vessels extracted by the regional connectivity method are sensitive to the starting position, the original images are rotated by $90^{\circ}, 180^{\circ}$, and $270^{\circ}$ to search for different starting points and initial blood vessel respectively. Finally, the vascular networks from different starting points are merged and a complete vascular network is obtained.

The third step is to optimize the obtained initial microvascular network. The binarized microvascular image can be obtained by the OTSU image binarization method ${ }^{18,19}$. In the initial vascular network, parts of the noise and artifacts could be marked as vessels due to their distance and gray value being close to real vessels. Thus we propose to apply the noise and artifacts reduction method into VCA, which is combined with the morphology and piece-by-piece analysis method. In this step, the skeleton of the microvascular in the OCTA image is extracted to obtain a thinner vessel graph which presents the vascular skeleton only. For further noise and artifacts reduction, a piece-by-piece analysis method is used to evaluate the correctness of extracted vascular skeleton. The branch and breakpoint information of each blood vessel curve can be used to obtain the branch length as well as total length of each blood vessel. If the branch length is too short, or the ratio of total length to the number of bifurcation points plus number of breakpoints is lower than the preset threshold, it will be considered as noise or artifacts for elimination. Accordingly, the noise and artifacts pixels can easily be distinguished from vessel pixels. A completely optimized vascular network is finally obtained after execution of all previous steps.

In the third part of further morphological characterization method, the length and width of vessels will be quantified. The total area $(S)$ and length $(L)$ of blood vessels are obtained through pixel accumulation 
from the vascular skeleton graphics, and the average width (W) of blood vessels can be obtained following indirect indices of mean trabecular plate thickness method ${ }^{20}$.

\section{Declarations}

\section{Ethics approval and consent to participate}

The trial has been registered with the Chinese Clinical Trial Registry (http://www.chictr.org/cn/ registration number: ChiCTR-IPR-17014160). The study has followed the tenets of the Declaration of Helsinki, and the study protocol has been approved by the Ethics Committee of First Affiliated Hospital of Nanjing Medical University (2015-SR-150). Informed written consents have been obtained from all patients after explaining possible consequences of the study.

\section{Consent for publication}

Not applicable.

\section{Availability of data and materials}

The datasets during and/or analysed during the current study available from the corresponding author on reasonable request.

\section{Competing interests}

The authors declare that they have no competing interests.

\section{Funding}

This work was supported by National Natural Science Foundation of China (nos. 11704188), the Natural Science Foundation of Jiangsu, China (no. BK 20170826), the Postdoctoral Science Foundation of China under grant no. 2019M651564, Beijing Bethune Charitable Foundation: BJ-LM2019002J.

\section{Authors' contributions}

SWW, WS, ZZH, and YS analyzed the patient data regarding the OCTA images. ZZH and YS acquired the OCTA images from the patients. WS, TF, LL, HF and YJX were the contributors in writing the manuscript. All authors read and approved the final manuscript.

\section{Acknowledgements}

Not applicable

\section{Abbreviations}

fibrovascular membrane (FVM), optical coherence tomography angiography (OCTA), 
proliferative diabetic retinopathy (PDR), vascular connectivity analysis (VCA),

diabetes mellitus (DM), vitreous hemorrhage $(\mathrm{VH})$, tractive retinal detachment (TRD),

fluorescein angiography (FA), intravitreal injection of conbercept (IVC).

\section{References}

1. Ning C, Mitchell P, Wong TY. Diabetic retinopathy. Lancet Lancet. 2010;376:124-36.

2. Amod, et al. Six-month visual outcome after pars plana vitrectomy in proliferative diabetic retinopathy with or without a single preoperative injection of intravitreal bevacizumab. International Ophthalmology (2012).

3. Chhablani D. Tyagi \& Narayanan Fluorescein angiography and optical coherence tomography in myopic choroidal neovascularization. Eye(2015).

4. Spaide, Richard F, PERIPHERAL AREAS OF, NONPERFUSION IN TREATED CENTRAL RETINAL VEIN OCCLUSION AS IMAGED BY WIDE-FIELD FLUORESCEIN ANGIOGRAPHY. Retina. 2011;31:829-37.

5. Zizhong, et al. OCT angiography-based monitoring of neovascular regression on fibrovascular membrane after preoperative intravitreal conbercept injection. Albrecht Von Graefes Archiv Fur Klinische Und Experimentelle Ophthalmologie (2019).

6. Cole ED, et al. Automated Ischemia Segmentation using OCT Angiography in Diabetic Retinopathy. Invest Ophthalmol Vis Sci. 2016;57:447-7.

7. Matsunaga D, Yi J, Puliafito CA, Kashani AH. OCT angiography in healthy human subjects. Ophthalmic Surg Lasers. 2014;45:510-5.

8. Chu S, Nesper PL, Soetikno BT, Bakri SJ, Fawzi AA. Projection-resolved OCT angiography of microvascular changes in paracentral acute middle maculopathy and acute macular neuroretinopathy. Invest Ophthalmol Vis Sci. 2018;59:2913-22.

9. Kim AY, et al. Quantifying Microvascular Density and Morphology in Diabetic Retinopathy Using Spectral-Domain Optical Coherence Tomography Angiography. Investigative Ophthalmology \& Visual Science 57 (2016).

10. Hwang TS, et al. Automated Quantification of Capillary Nonperfusion Using Optical Coherence Tomography Angiography in Diabetic Retinopathy. JAMA Ophthalmology. 2016;134:367-73.

11. Poole KM, Patil CA, Nelson CE, Mccormack DR, Skala MC Longitudinal study of arteriogenesis with swept source optical coherence tomography and hyperspectral imaging. Proceedings of SPIE - The International Society for Optical Engineering 8934 (2014).

12. Kowalski WJ, Teslovich NC, Pekkan K, Chen CY, Keller BB Simultaneous real-time quantification of blood flow and vascular growth in the chick embryo using optical coherence tomography. Proceedings of Spie the International Society for Optical Engineering 8953, 121-134 (2014).

13. Yousefi S, Liu T, Wang RK. Segmentation and quantification of blood vessels for OCT-based microangiograms using hybrid shape/intensity compounding. Microvasc Res. 2015;97:37-46. 
14. Choi W, Mohler KJ, Potsaid BM, Chen DL, Fujimoto JG. Choriocapillaris and Choroidal Microvasculature Imaging with Ultrahigh Speed OCT Angiography. Plos One. 2013;8:e81499.

15. He J, et al. Segmentation of diabetic macular edema in OCT retinal images. Opto-Electronic Engineering (2018).

16. Himaga M, Usher D, Boyce JF. Accurate Retinal Blood Vessel Segmentation by Using Multi-Resolution Matched Filtering and Directional Region Growing. leice Transinf Syst. 2004;87-D:155-63.

17. Chanwimaluang T, Fan G in International Symposium on Circuits \& Systems (2003).

18. Qiu L, Xuan Z, Zhang X. Debris Color Image Segmentaion by K-means Clustering and Ostu Method. Lubrication Engineering (2014).

19. Shi W, Li F, Chen S, University C. Algorithm for Image Binarization Based on Improved Otsu and Niblack. World Sci-Tech R \& D(2013).

20. Parfitt M. Villanueva \& Kleerekoper Relationships between surface, volume, and thickness of iliac trabecular bone in aging and in osteoporosis. Implications for the microanatomic and cellular mechanisms of bone loss. Journal of Clinical Investigation (1983).

\section{Figures}
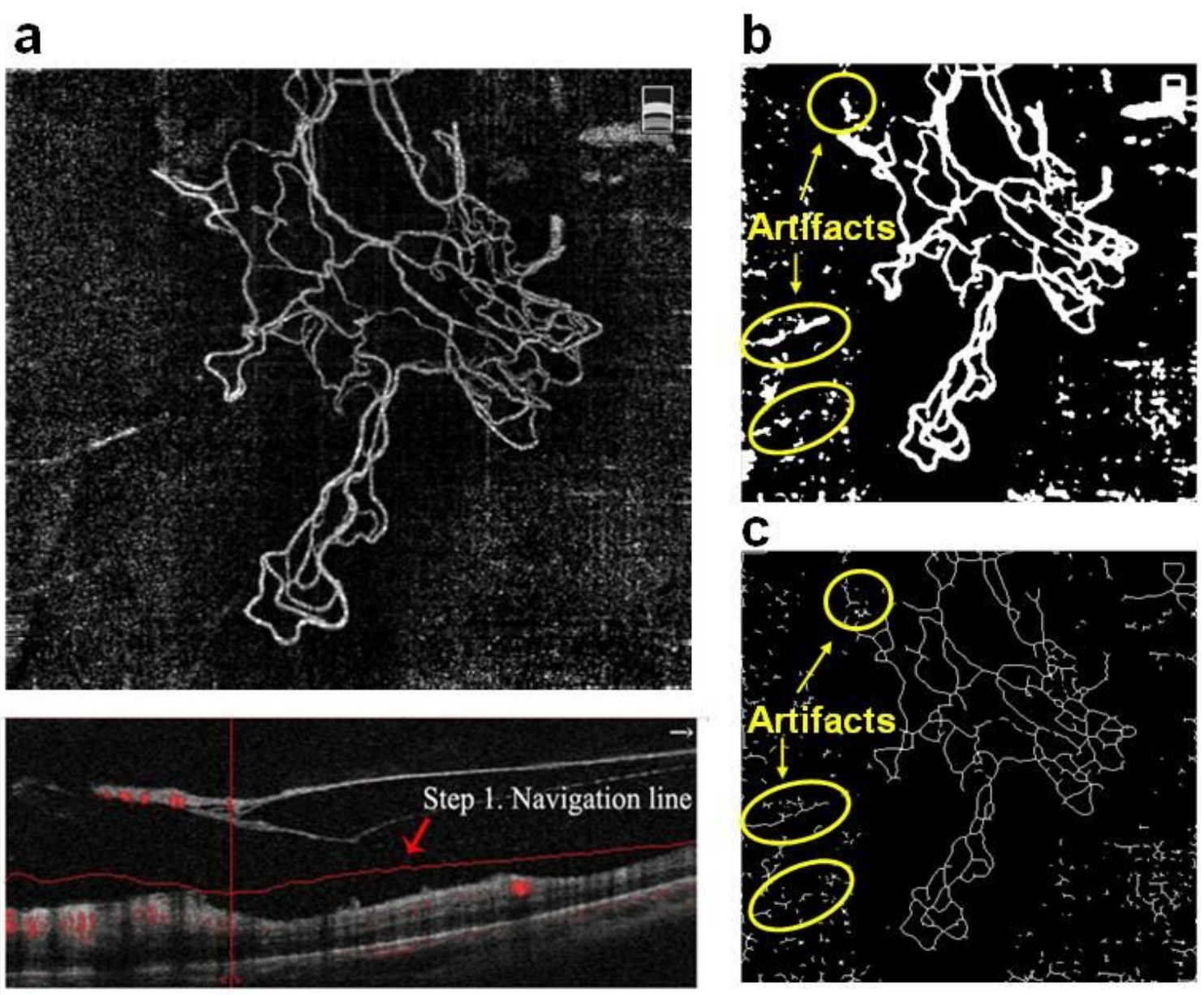
Figure 1

(a) Original images of the neovascularization on FVM floating in the vitreous cavity. (b) Vessel extraction resulted from the matched filter algorithm. (c) The skeleton of vessel of image (b) obtained by the skeleton extraction algorithm.
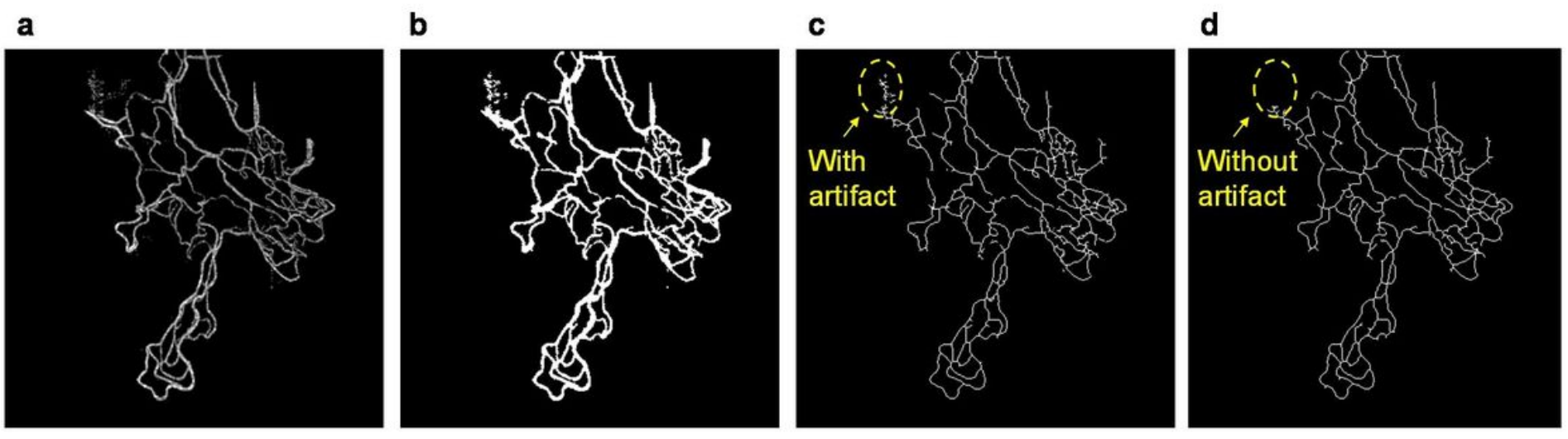

Figure 2

(a) The extraction of vascular network with the improved VCA method. (b) Binarized image of (a). (c) The skeleton of the vascular network. (d) A noise-free complete skeleton of the blood vessel by the piece-bypiece analysis method. 
b
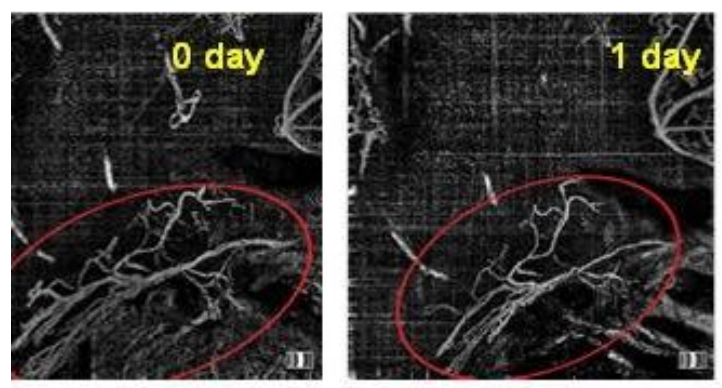

g
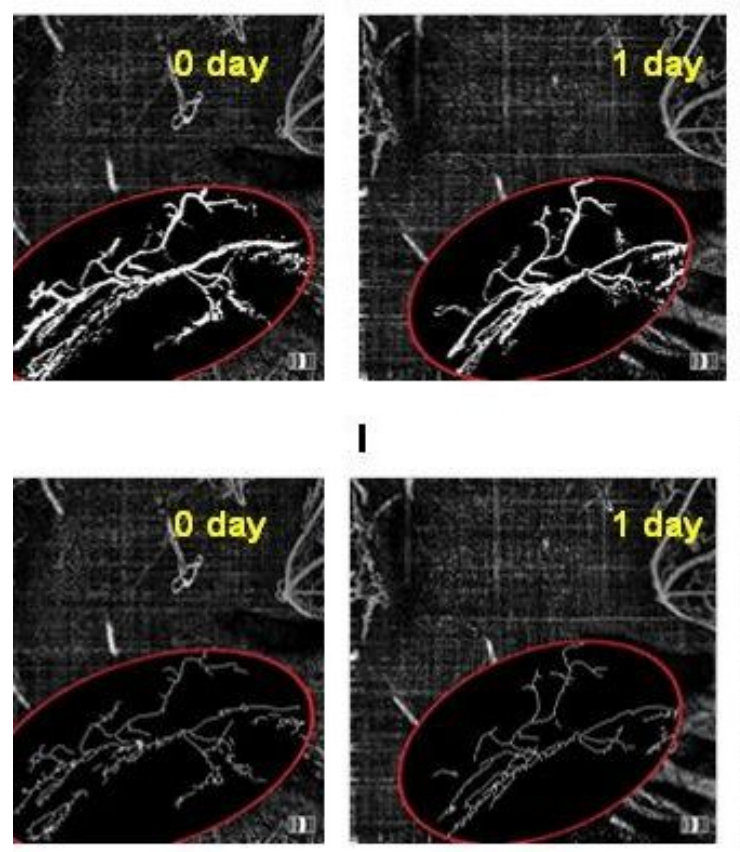

c

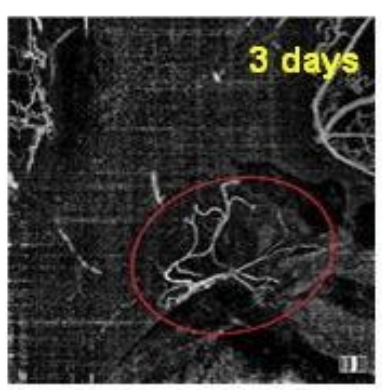

h

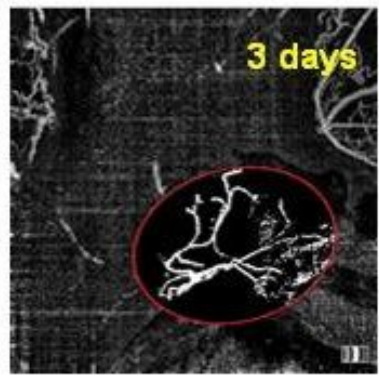

m

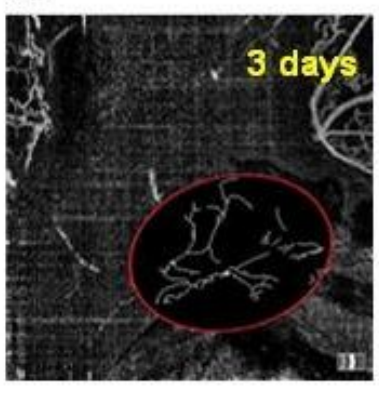

d

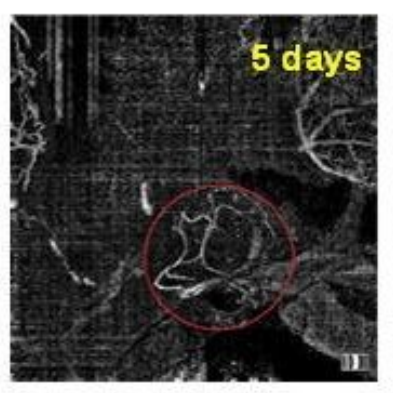

i
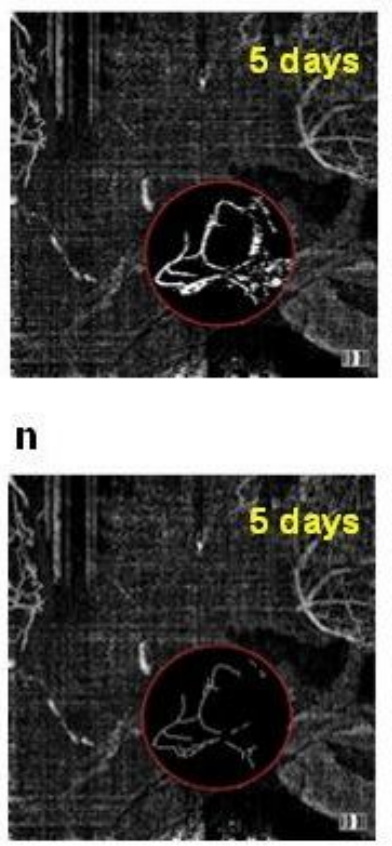

e

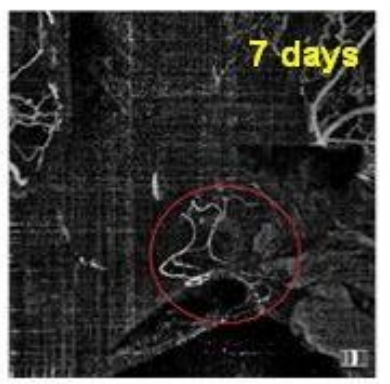

j

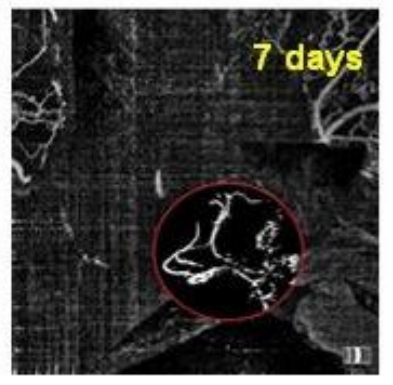

o

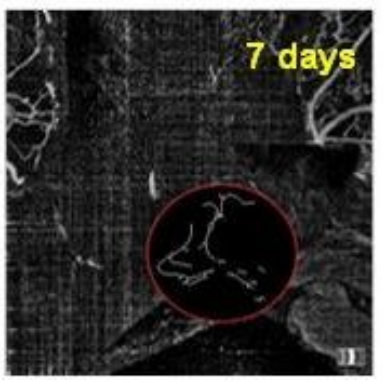

Figure 3

(a) Distribution of the neovascularization on FVM of the patient before treatment. (b-e) Proliferation of the same region of the patient during treatment stages $(1,3,5,7$ days respectively). ( $f-j)$ The binarized QCTA image results after the improved VCA for the neovascularization shown in (a-e). (k-o) The corresponding vascular skeleton extraction results for the neovascularization shown in (a-e). 
a

\begin{tabular}{|cccc|}
\hline No. & $\begin{array}{c}\text { Treated } \\
\text { time(days) }\end{array}$ & Length (px) & Width (px) \\
\hline 1 & 0 & 2512 & 2.9 \\
\hline 2 & 1 & 2006 & 3.0 \\
\hline 3 & 3 & 1172 & 3.3 \\
\hline 4 & 5 & 618 & 4.1 \\
\hline 5 & 7 & 557 & 4.6 \\
\hline
\end{tabular}

b

C

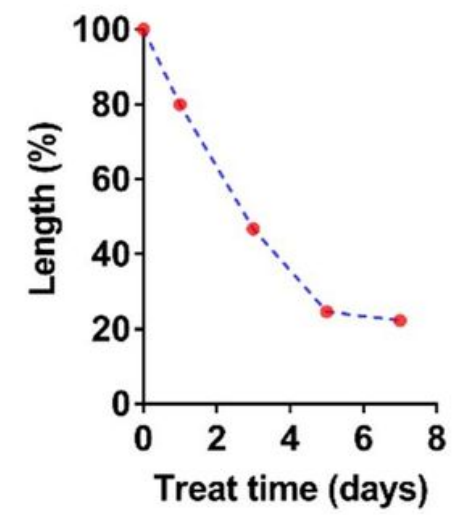

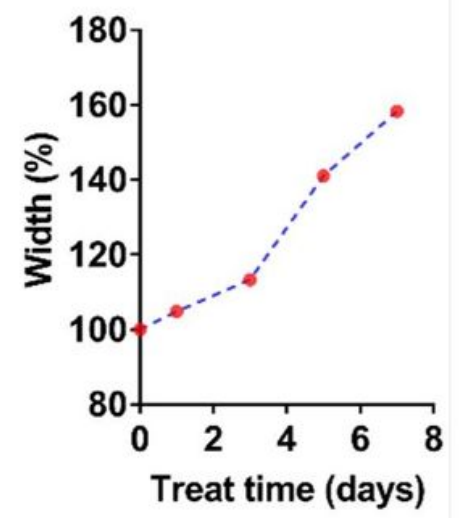

Figure 4

(a) Quantified results of the treatment monitoring of neovascularization on FVM from a patient with proliferative diabetic retinopathy (PDR). (b) The quantified length of the vessel as a function of treatment duration. (c) The quantified width of the vessel as a function of treatment duration.

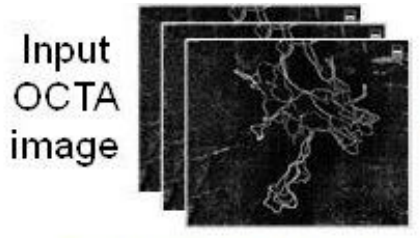

Pre-processing

Image cropping (ROI selection) $\rightarrow$ Color space conversion

Vessel extracting

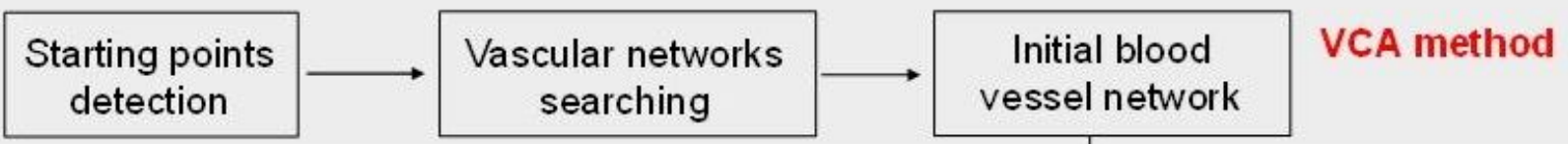

Skeleton extraction of the blood vessel

Binarization by automatic

VCA improvement
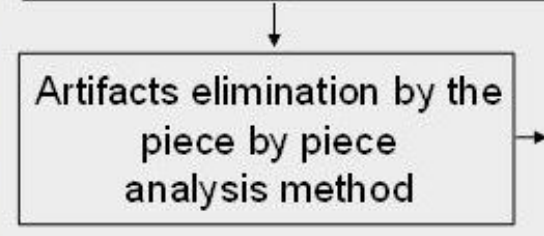

Detected blood vessel network thresholding OSTU method

\begin{tabular}{c|c} 
Detected \\
blood vessel \\
network
\end{tabular}$\rightarrow \rightarrow \begin{gathered}\text { Merging the network } \\
\text { with different } \\
\text { starting points }\end{gathered}$

Final vessel segment image
Vessel quantification

\begin{tabular}{|c|} 
Length \\
quantification
\end{tabular}$\longrightarrow$\begin{tabular}{c|} 
Width \\
quantification
\end{tabular}

Output of the extracted vessels and quantified results 
Figure 5

The flowchart of proposed optimization method. 